\title{
Domain wall network as QCD vacuum: confinement, chiral symmetry, hadronization
}

\author{
Sergei N. Nedelko ${ }^{1, a}$ and Vladimir V. Voronin ${ }^{1}$ \\ ${ }^{1}$ Bogoliubov Laboratory of Theoretical Physics, JINR, Dubna, Russia
}

\begin{abstract}
An approach to QCD vacuum as a medium describable in terms of statistical ensemble of almost everywhere homogeneous Abelian (anti-)self-dual gluon fields is reviewed. These fields play the role of the confining medium for color charged fields as well as underline the mechanism of realization of chiral $S U_{\mathrm{L}}\left(N_{f}\right) \times S U_{\mathrm{R}}\left(N_{f}\right)$ and $U_{\mathrm{A}}(1)$ symmetries. Hadronization formalism based on this ensemble leads to manifestly defined quantum effective meson action. Strong, electromagnetic and weak interactions of mesons are represented in the action in terms of nonlocal $n$-point interaction vertices given by the quark-gluon loops averaged over the background ensemble. Systematic results for the mass spectrum and decay constants of radially excited light, heavy-light mesons and heavy quarkonia are presented. Relationship of this approach to the results of functional renormalization group and Dyson-Schwinger equations, and the picture of harmonic confinement is briefly outlined.
\end{abstract}

\section{Introduction}

The idea of four-dimensional harmonic oscillator as a tool for universal description of the Regge spectrum of hadron masses was formulated long time ago by Feynman, Kislinger, and Ravndal [1] and later re-entered the discussion about quark confinement several times in various ways. In particular, Leutwyler and Stern developed the formalism devoted to the covariant description of bilocal mesonlike fields combined with the idea of harmonic confinement [2-6]. In recent years, the approaches to confinement based on the soft wall AdS/QCD model with harmonic potential demonstrated an impressive phenomenological success.

The approach to QCD vacuum and hadronization presented here has been developed in a series of papers [7-13]. It clearly incorporates the idea of harmonic confinement both in terms of elementary color charged fields and the composite colorless hadron fields. The distinctive feature of the approach is that it links the concept of harmonic confinement and Regge character of hadron mass spectrum to the specific class of nonperturbative gluon configurations - almost everywhere homogeneous Abelian (anti-)self-dual gluon fields. A close interrelation of the Abelian (anti-)self-dual fields and the hadronization based on harmonic confinement can be read off the papers [2-6, 14-17]. In brief, the line of arguments is as follows (for more detailed exposition see [12, 13]).

An important starting point is due to the observation of Pagels and Tomboulis [17] that Abelian self-dual fields describe a medium that is infinitely stiff to small gauge field fluctuations, i.e. the wave

\footnotetext{
a e-mail: nedelko@theor.jinr.ru
} 


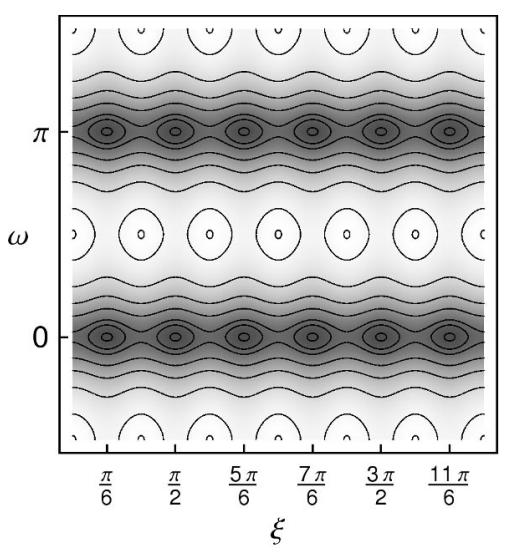

Figure 1. Effective potential as a function of the angle $\omega$ between chromomagnetic and chromoelectric field and the mixing angle $\xi$ in the Cartan subalgebra. The minima in the dark gray regions correspond to the Abelian (anti-)self-dual configurations and form a periodic structure labelled by integer indices $(k l)$ in Eq. (1) (for more details see $[9,12,20])$.

solutions for the effective quantum equations of motion are absent. This feature was interpreted as suggestive of confinement of color. Strong argumentation in favour of the Abelian (anti-)self-dual homogeneous field as a candidate for the global nontrivial minimum of the effective action originates from the papers $[14,16,19-21]$. Leutwyler has shown that the constant gauge field is stable against small quantum fluctuations only if it is Abelian (anti-)self-dual covariantly constant field [14, 16]. Nonperturbative calculation of the effective potential within the functional renormalization group [19] supported the earlier one-loop results on existence of the nontrivial minimum of the effective action for the Abelian (anti-)self-dual field.

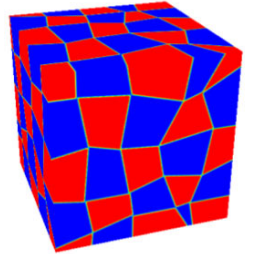

$\left\langle F^{2}\right\rangle=B^{2}$

$\langle|F \tilde{F}|\rangle=B^{2}$

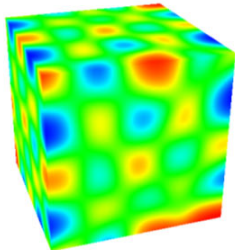

$\left\langle F^{2}\right\rangle=B^{2}$ $\langle|F \tilde{F}|\rangle \ll B^{2}$
Figure 2. Topological charge density for domain wall networks with different values of the wall width. The left picture is an example of confining almost everywhere homogeneous Abelian (anti-)self-dual fields. Red (blue) color corresponds to the self-dual field (anti-self-dual), green - pure chromomagnetic field. The right plot represents the case of preferably pure chromomagnetic field when the topological charge density is nearly zero and color charged quasiparticles can be excited thus indicating deconfinement (for more details see [12]).

The eigenvalues of the Dirac and Klein-Gordon operators in the presence of Abelian self-dual field are purely discrete, and the corresponding eigenfunctions of quarks and gluons charged with respect to the background are of the bound state type. This is a consequence of the fact that these operators contain the four-dimensional harmonic oscillator. Eigenmodes of the color charged fields have no (quasi-)particle interpretation but describe field fluctuations decaying in space and time. The consequence of this property is that the momentum representation of the translation invariant part of the propagator of the color charged field in the background of homogeneous (anti-)self-dual Abelian gauge field is an entire analytical function. The absence of pole in the propagator was treated as the absence of the particle interpretation of the charged field [15]. However, neither the homogeneous Abelian (anti-)self-dual field itself nor the form of gluon propagator in the presence of this background had the clue to linear quark-antiquark potential. Nevertheless, the analytic structure of the gluon and quark propagators and assumption about the randomness of the background field ensemble led both to the area law for static quarks and the Regge spectrum for light hadrons.

The model of hadronization developed in $[7,8,12,13]$ indicated that the spectrum of mesons displayed the Regge character both with respect to total angular momentum and radial quantum number 
of the meson. The reason for confinement of a single quark and Regge spectrum of mesons turned out to be the same - the analytic properties of quark and gluon propagators. In this formalism any meson looks much more like a complicated collective excitation of a medium (QCD vacuum) involving quark, antiquark and gluon fields than a nonrelativistic quantum mechanical bound state of charged particles (quark and anti-quark) due to some potential interaction between them. Within this relativistic quantum field description the Regge spectrum of color neutral collective modes appeared as a "medium effect" as well as the suppression (confinement) of a color charged elementary modes.

These observations have almost completed the quark confinement picture based on the random almost everywhere homogeneous Abelian (anti-)self-dual fields. Self-duality of the fields plays the crucial role in this picture. This random field ensemble represents a medium where the color charged elementary excitations exist as quickly decaying in space and time field fluctuations but the collective colorless excitations (mesons) can propagate as plain waves (particles). Besides this dynamical color charge confinement, a correct complete picture must include the limit of static quark-antiquark pair with the area law for the temporal Wilson loop. In order to explore this aspect an explicit construction of the random domain ensemble was suggested in paper [9], and the area law for the Wilson loop was demonstrated by the explicit calculation. Randomness of the ensemble (in line with [22]) and (anti-)self-duality of the fields are crucial for this result.

The character of meson wave functions in hadronization approach [8] is fixed by the form of the gluon propagator in the background of specific vacuum gluon configurations. These wave functions are very similar to the wave functions of the soft wall AdS/QCD with quadratic dilaton profile and Leutwyler-Stern formalism. In all three cases one deals with the generalized Laguerre polynomials as characteristic for the radial meson excitations. Another interesting observation is that the form of Euclidean gluon and quark propagators in the presence of the Abelian (anti-)self-dual background are in qualitative agreement with the decoupling scenario of the infra-red behaviour of the propagators in Dyson-Schwinger equations (DSE) and functional renormalization group (FRG) approaches and lattice QCD results for the Landau gauge propagators.

The next section is devoted to motivation of the approach based on the domain wall network gluon configurations. xt of the spontaneous chiral symmetry breaking by the background field and four-fermion interaction. The structure of the effective meson action and the results for the masses, transition and decay constants of various mesons are presented in section 3 . In the last section we outline the relation of gluon propagator in the model under consideration to the results of FRG and DSE.

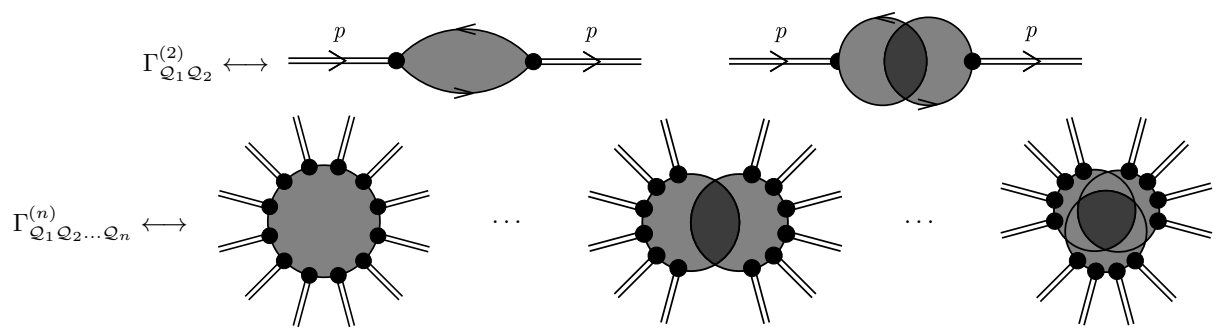

Figure 3. Diagrammatic representation of nonlocal meson vertex functions. Light grey denotes averaging over background field, dark grey denotes correlation of loop diagrams by background field. 


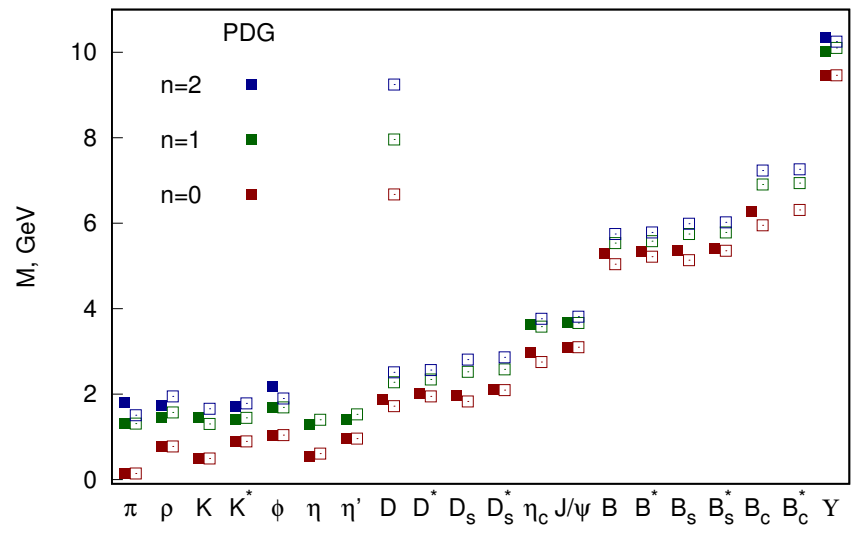

Figure 4. The masses of various radially excited mesons. The same values of parameters were used for all mesons shown in the figure.

\section{Scalar gluon condensate and the effective action of QCD}

The phenomenological basis of the present approach is the assumption about existence of nonzero gluon condensates in QCD, first of all - the scalar condensate $\left\langle g^{2} F^{2}\right\rangle$. In order to incorporate this condensate into the functional integral approach to quantization of QCD one has to choose appropriate conditions for the functional space of gluon fields $A_{\mu}^{a}$ to be integrated over (see, e.g., Ref.[18]). Besides the formal mathematical content, these conditions play the role of substantial physical input which, together with the classical action of QCD, complements the statement of the quantization problem. In other words, starting with the very basic representation of the Euclidean functional integral for QCD, one has to specify integration spaces $\mathcal{F}_{A}$ for gluon and $\Psi$ for quark fields. Bearing in mind a nontrivial QCD vacuum structure encoded in various condensates, one have to define $\mathcal{F}_{A}$ permitting gluon fields with nonzero classical action density. The gauge fields $A$ that satisfy this condition have a potential to provide the vacuum with the whole variety of condensates.

An analytical approach to definition and calculation of the functional integral can be based on separation of gluon modes $B_{\mu}^{a}$ responsible for nonzero condensates from the small perturbations $Q_{\mu}^{a}$. This separation must be supplemented with gauge fixing. Background gauge fixing condition $D(B) Q=0$ is the most natural choice. To perform separation, one inserts identity

$$
1=\int_{\mathcal{B}} D B \Phi[A, B] \int_{Q} D Q \int_{\Omega} D \omega \delta\left[A^{\omega}-Q^{\omega}-B^{\omega}\right] \delta\left[D\left(B^{\omega}\right) Q^{\omega}\right]
$$

in the functional integral and arrives at

$$
\begin{aligned}
Z & =N^{\prime} \int_{\mathcal{B}} D B \int_{\Psi} D \psi D \bar{\psi} \int_{Q} D Q \operatorname{det}[\mathcal{D}(B) \mathcal{D}(B+Q)] \delta[\mathcal{D}(B) Q] e^{-S_{\mathrm{QCD}}[B+Q, \psi, \bar{\psi}]} \\
& =\int_{\mathcal{B}} D B \exp \left\{-S_{\mathrm{eff}}[B]\right\} .
\end{aligned}
$$

Thus defined quantum effective action $S_{\text {eff }}[B]$ has a physical meaning of the free energy of the quantum field system in the presence of the background gluon field $B_{\mu}^{a}$. In the limit $V \rightarrow \infty$ global minima of $S_{\text {eff }}[B]$ determine the class of gauge field configurations representing the equilibrium state (vacuum) of the system. Quite reliable argumentation in favour of (almost everywhere) homogeneous Abelian (anti-)self-dual fields as dominating vacuum configurations in pure gluodynamics was put 
Table 1. Model parameters fitted to the masses of $\pi, \rho, K, K, \eta, J / \psi, \Upsilon$ and used in calculation of all other meson masses, decay and transition constants.

\begin{tabular}{ccccccc}
\hline$m_{u / d}, \mathrm{MeV}$ & $m_{s}, \mathrm{MeV}$ & $m_{c}, \mathrm{MeV}$ & $m_{b}, \mathrm{MeV}$ & $\Lambda, \mathrm{MeV}$ & $\alpha_{s}$ & $R, \mathrm{fm}$ \\
\hline 145 & 376 & 1566 & 4879 & 416 & 3.45 & 1.12 \\
\hline
\end{tabular}

forward by many authors $[14,17]$. As it has already been mentioned in Introduction, nonperturbative calculation of QCD quantum effective action within the functional renormalization group approach [19] supported the one-loop result $[14,16,17]$ and indicated the existence of a minimum of the effective potential for nonzero value of Abelian (anti-)self-dual homogeneous gluon field.

Table 2. Decay and transition constants of various pseudoscalar and vector mesons calculated through the diagrams shown in Figs. 5 and 6.

\begin{tabular}{cccc|cccc}
\hline Meson & $n$ & $\begin{array}{c}f_{P}^{\mathrm{exp}} \\
(\mathrm{MeV})\end{array}$ & $\begin{array}{c}f_{P} \\
(\mathrm{MeV})\end{array}$ & Meson & $n$ & $g_{V \gamma}[23]$ & $g_{V \gamma}$ \\
\hline$\pi$ & 0 & $130[23]$ & 140 & $\rho$ & 0 & 0.2 & 0.2 \\
$\pi(1300)$ & 1 & & 29 & $\rho$ & 1 & & 0.053 \\
\hline$K$ & 0 & $156[23]$ & 175 & $\omega$ & 0 & 0.059 & 0.067 \\
$K(1460)$ & 1 & & 27 & $\omega$ & 1 & & 0.018 \\
\hline$D$ & 0 & $205[23]$ & 212 & $\phi$ & 0 & 0.074 & 0.071 \\
$D$ & 1 & & 51 & $\phi$ & 1 & & 0.02 \\
\hline$D_{s}$ & 0 & $258[23]$ & 274 & $J / \psi$ & 0 & 0.09 & 0.06 \\
$D_{s}$ & 1 & & 57 & $J / \psi$ & 1 & & 0.015 \\
\hline$B$ & 0 & $191[23]$ & 187 & $\Upsilon$ & 0 & 0.025 & 0.014 \\
$B$ & 1 & & 55 & $\Upsilon$ & 1 & & 0.0019 \\
\hline$B_{s}$ & 0 & $253[24]$ & 248 & & & & \\
$B_{s}$ & 1 & & 68 & & & & \\
\hline$B_{c}$ & 0 & $489[24]$ & 434 & & & & \\
$B_{c}$ & 1 & & 135 & & & & \\
\hline
\end{tabular}

Ginzburg-Landau (GL) approach to the quantum effective action indicated a possibility of the domain wall network formation in QCD vacuum resulting in the dominating vacuum gluon configuration seen as an ensemble of densely packed lumps of covariantly constant Abelian (anti-)self-dual field $[9,12,20,21]$. Nonzero scalar gluon condensate $\left\langle g^{2} F_{\mu \nu}^{a} F_{\mu \nu}^{a}\right\rangle$ postulated by the effective potential leads to the existence of twelve discrete degenerate global minima of the effective action (see Fig.1),

$$
\begin{aligned}
& \breve{A}_{\mu} \in\left\{\breve{B}_{\mu}^{(k l)} \mid k=0,1, \ldots, 5 ; l=0,1\right\}, \breve{B}_{\mu}^{(k l)}=-\frac{1}{2} \breve{n}_{k} B_{\mu \nu}^{(l)} x_{v}, \\
& \tilde{B}_{\mu \nu}^{(l)}=\frac{1}{2} \varepsilon_{\mu \nu \alpha \beta} B_{\alpha \beta}^{(l)}=(-1)^{l} B_{\mu \nu}^{(l)}, \breve{n}_{k}=T^{3} \cos \left(\xi_{k}\right)+T^{8} \sin \left(\xi_{k}\right), \quad \xi_{k}=\frac{2 k+1}{6} \pi,
\end{aligned}
$$

where $l=0$ and $l=1$ correspond to the self-dual and anti-self-dual field respectively, matrix $\breve{n}_{k}$ belongs to Cartan subalgebra of $s u(3)$ with six values of the angle $\xi_{k}$ corresponding to the boundaries of the Weyl chambers in the root space of $s u(3)$. The minima are connected by the parity and Weyl group reflections. Their existence indicates that the system is prone to the domain wall formation. To demonstrate the simplest example of domain wall interpolating between the self-dual and anti-selfdual Abelian configurations, one allows the angle $\omega$ between chromomagnetic and chromoelectric fields to vary from point to point in $R^{4}$ and restricts other degrees of freedom of gluon field to their 




(a)

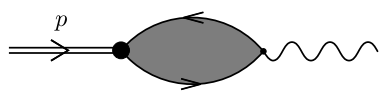

(a)

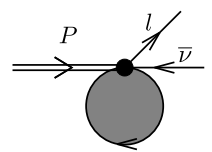

(b)

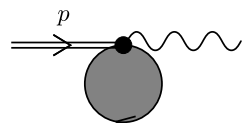

(b)
Figure 5. Diagrams contributing to leptonic decay constants $f_{P}$.

Figure 6. Diagrams contributing to $V \rightarrow \gamma$ transition constants $g_{V \gamma}$.

vacuum values. In this case Ginsburg-Landau Lagrangian leads to the sine-Gordon equation for $\omega$ with the standard kink solution (for details see Ref. $[12,20]$ ). Away from the kink location vacuum field is almost self-dual $(\omega=0)$ or anti-self-dual $(\omega=\pi)$. Exactly at the wall it becomes purely chromomagnetic $(\omega=\pi / 2)$. Domain wall network is constructed by means of the kink superposition. Topological charge density distribution for a network of domain walls with different width is illustrated in Fig.2.

Based on this construction, the measure of integration over the background field $B_{\mu}^{a}$ can be constructively represented as the infinite dimensional (in the infinite volume) integral over the parameters of $N \rightarrow \infty$ domain walls in the network: their positions, orientations and widths, with the weight determined by the effective action. The explicit construction of the domain wall network is the most recent development of the formalism that have been studied in the series of papers [7-11], in which the domain wall defects in the homogeneous Abelian (anti-)self-dual field were taken into account either implicitly or in an explicit but simplified form with the spherical domains. The practical calculations in the next sections will be done within combined implementation of domain model given in paper [10]: propagators in the quark loops are taken in the approximation of the homogeneous background field and the quark loops are averaged over the background field, the correlators of the background field are calculated in the spherical domain approximation.

\section{Meson properties}

The truncated QCD functional integral can be rewritten in terms of the composite colorless meson fields $\phi_{Q}$ by means of the standard bosonization procedure: introduce the auxiliary meson fields, integrate out the quark fields, perform the orthogonal transformation of the auxiliary fields that diagonalizes the quadratic part of the action and, finally, rescale the meson fields to provide the correct residue of the meson propagator at the pole corresponding to its physical mass (if any). More details can be found in Ref. $[7,8,10]$. The result can be written in the following compact form

$$
\begin{aligned}
& Z=\mathcal{N}[13] \int D \phi_{Q} \exp \left\{-\frac{\Lambda^{2}}{2} \frac{h_{Q}^{2}}{g^{2} C_{Q}^{2}} \int d^{4} x \phi_{Q}^{2}(x)-\sum_{k=2}^{\infty} \frac{1}{k} W_{k}[\phi]\right\}, \\
& W_{k}[\phi]=\sum_{Q_{1} \ldots Q_{k}} h_{Q_{1}} \ldots h_{Q_{k}} \int d^{4} x_{1} \ldots \int d^{4} x_{k} \Phi_{Q_{1}}\left(x_{1}\right) \ldots \Phi_{Q_{k}}\left(x_{k}\right) \Gamma_{Q_{1} \ldots Q_{k}}^{(k)}\left(x_{1}, \ldots, x_{k}\right), \\
& \Phi_{Q}(x)=\int \frac{d^{4} p}{(2 \pi)^{4}} e^{i p x} O_{Q Q^{\prime}}(p) \tilde{\phi}_{Q^{\prime}}(p), .
\end{aligned}
$$

where condensed index $Q$ denotes all relevant meson quantum numbers and indices. Integration variables $\phi_{Q}$ in the functional integral (2) correspond to the physical meson fields that diagonalize the 


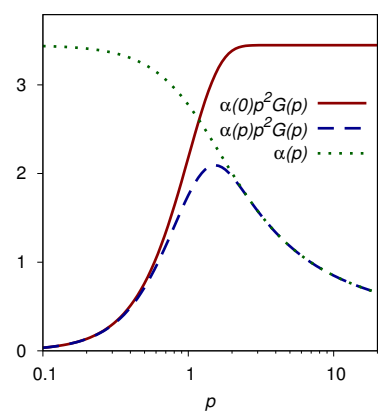

Figure 7. Momentum dependence of the gluon dressing function without (solid line) and with (dashed line) accounting for the running of the strong coupling constant $\alpha_{s}(p)$ (dotted line). The dashed line qualitatively reproduces the shape of the Landau gauge dressing function of gluons calculated within the functional renormalization group [25] and Lattice QCD [26, 27] as well as a part of the input gluon propagator used in the approaches based on combined Dyson-Schwinger and Bethe-Salpeter equations [28, 29].

quadratic part of the effective meson action (3) in momentum representation, which is achieved by means of orthogonal transformation $O(p)$.

Interactions between physical meson fields $\phi_{Q}$ are described by $k$-point nonlocal vertices $\Gamma_{Q_{1} \ldots Q_{2}}^{(k)}$. subsequently tuned to the physical meson representation by means of corresponding orthogonal transformations $O(p)$. As is illustrated in Fig. 3, vertices $\Gamma^{(k)}$ are expressed via 1-loop diagrams $G_{Q_{1} \ldots Q_{k}}^{(k)}$ which include nonlocal quark-meson vertices and quark propagators (for their explicit analytical form see [13]).

The mass spectrum $M_{Q}$ of mesons and quark-meson coupling constants $h_{Q}$ are determined by the quadratic part of the effective meson action via equations

$$
1=\frac{g^{2}}{\Lambda^{2}} C_{Q}^{2} \tilde{\Pi}_{Q}\left(-M_{Q}^{2} \mid B\right), \quad h_{Q}^{-2}=\left.\frac{d}{d p^{2}} \tilde{\Pi}_{Q}\left(p^{2}\right)\right|_{p^{2}=-M_{Q}^{2}},
$$

where $\tilde{\Pi}_{Q}\left(p^{2}\right)$ is the diagonalized two-point correlator $\tilde{\Gamma}_{Q Q^{\prime}}^{(2)}(p)$ put on mass shell. Above definition of the meson-quark coupling constant $h_{Q}$ provides correct residue at the pole.

The results of calculations are shown in Fig. 4 and Table 2. An overall accuracy of description is 10-15 percent in the lowest order calculation achieved with the minimal for QCD set of parameters: infrared limits of renormalized strong coupling constant $\alpha_{s}$ and quark masses $m_{f}$, scalar gluon condensate $\left\langle g^{2} F^{2}\right\rangle$ as a fundamental scale of QCD and topological susceptibility of pure QCD without quarks. This last parameter can be related to the mean size $R$ of domains.

\section{Discussion}

It is interesting to take a look at the properties of gluon propagator in the present approach in view of the known functional form of quark and Landau gauge gluon propagators calculated within the functional renormalization group, Lattice QCD and Dyson-Schwinger equations [25-29]. As it can be seen from Fig. 7 the shape of the gluon dressing function in the background field under consideration is in qualitative agreement with the known results of functional RG, DSE and lattice QCD. For more detailed comparison we refer to the paper [13].

For conclusion we would like to outline few problems to be addressed within the domain model of QCD vacuum. The most important conceptual question relates to identification of a mechanism for stabilization of a finite mean size of domains. Our preliminary estimates indicated that the competition between gluon and ghost contributions and the contribution of the quark lowest eigenvalues to the free energy density of the finite domain could lead to the appropriate stabilization. This issue has to be studied carefully. Another important for phenomenological applications problem is the accurate description of the $n$-pont correlators in the random domain wall network ensemble which can be 
achieved by numerical methods. Random spherical domain ensemble is a rather rough approximation, and it has to be improved. Domain model of QCD vacuum offers an appealing way to study the deconfinement transition in terms of the explicit degrees of freedom which are active or suppressed in different regimes (high energy density, high baryon charge density, strong electromagnetic fields). A preliminary consideration of the relevant features of the model can be found in [12].

\section{References}

[1] R. P. Feynman, M, Kislinger, and F. Ravndal, Phys. Rev. D 3 (1971) 2706.

[2] H. Leutwyler and J. Stern, Phys. Lett. B 73, 75 (1978).

[3] H. Leutwyler and J. Stern, Annals Phys. 112, 94 (1978).

[4] H. Leutwyler and J. Stern, Phys. Lett. B 69, 207 (1977).

[5] H. Leutwyler and J. Stern, Nucl. Phys. B 133, 115 (1978).

[6] H. Leutwyler and J. Stern, Nucl. Phys. B 157, 327 (1979).

[7] G.V. Efimov, and S.N. Nedelko, Phys. Rev. D 51, 176 (1995).

[8] J. V. Burdanov, G. V. Efimov, S. N. Nedelko, S. A. Solunin, Phys. Rev. D 54, 4483 (1996).

[9] A.C. Kalloniatis and S.N. Nedelko, Phys. Rev. D 64, 114025 (2001).

[10] A.C. Kalloniatis and S.N. Nedelko, Phys. Rev. D 69, 074029 (2004).

[11] A. C. Kalloniatis and S. N. Nedelko, Phys. Rev. D 73, 034006 (2006).

[12] S. N. Nedelko and V. E. Voronin, Eur. Phys. J. A 51, 45 (2015).

[13] S. N. Nedelko and V. E. Voronin, Phys. Rev. D 93, 094010 (2016).

[14] H. Leutwyler, Nucl. Phys. B 179 , 129 (1981).

[15] H. Leutwyler, Phys. Lett. B 96, 154 (1980).

[16] P. Minkowski, Phys. Lett. B 76, 439 (1978).

[17] H. Pagels, and E. Tomboulis, Nucl. Phys. B 143, 485 (1978).

[18] L.D. Faddeev, arXiv:0911.1013 [math-ph].

[19] A. Eichhorn, H. Gies and J. M. Pawlowski, Phys. Rev. D 83, 045014 (2011).

[20] B.V. Galilo and S.N. Nedelko, Phys. Part. Nucl. Lett., 8, 67 (2011).

[21] D. P. George, A. Ram, J. E. Thompson and R. R. Volkas, Phys. Rev. D 87, 105009 (2013). [arXiv:1203.1048 [hep-th]].

[22] P. Olesen, Nucl. Phys. B 200, 381 (1982).

[23] K.A. Olive et al. (Particle Data Group) Chinese Phys. C 38,090001 (2014).

[24] T. W. Chiu et al. [TWQCD Collaboration], PoS LAT 2006, 180 (2007).

[25] M. Mitter, J. M. Pawlowski and N. Strodthoff, Phys. Rev. D 91054035 (2015).

[26] P. O. Bowman, U. M. Heller, D. B. Leinweber, M. B. Parappilly and A. G. Williams, Phys. Rev. D 70, (2004) 034509; [hep-lat/0402032].

[27] A. Sternbeck, E.-M. Ilgenfritz, M. Muller-Preussker, A. Schiller and I. L. Bogolubsky, PoS LAT 2006, 076 (2006). [hep-lat/0610053].

[28] C. S. Fischer, S. Kubrak and R. Williams, Eur. Phys. J. A 50, 126 (2014).

[29] S. M. Dorkin, L. P. Kaptari and B. Kämpfer, Phys. Rev. C 91 (2015) 055201, arXiv:1412.3345 [hep-ph]. 